\title{
Statistische Zählsprengel und ihre Anwendung in der thematischen Kartographie
}

\author{
Martin Weber \\ Österreichisches Statistisches Zentralamt, Wien
}

\begin{abstract}
Zusammenfassung: Die Statistischen Zählsprengel stellen die kleinsten räumlichen Einheiten der amtlichen Statistik dar, über die Großzählungsdaten verfügbar sind. Bei den Statistischen Zählsprengeln tritt das Problem der räumlichen Vergleichbarkeit eines Sachverhaltes auf, da die räumlichen Bezugsflächen in ihrer Größe stark differieren. Eine Möglichkeit der Gebietsgliederung in möglichst gleich große Bezugsflächen ist die Verwendung von Quadratgitternetzen. Dabei werden die Siedlungsschwerpunkte der Zählsprengel möglichst kleinen Gitternetzmaschen zugeordnet und die Daten aggregiert.
\end{abstract}

\begin{abstract}
The enumeration districts are the smallest territorial units of official statistics for which data of the combined census are available. Considering the enumeration districts there exists the problem of the territorial comparability of a state of facts as the reference areas differ widely in their size. A possibility of a spacial breakdown into reference areas of as possible the same size is the application of square grid nets. Thereby the settlement centres of gravity of the enumeration districts are related to grid meshes which are as small as possible and the data are aggregated.
\end{abstract}

\section{Problemstellung}

Bei der kartographischen Aufbereitung der statistischen Großzählungsdaten stellt sich die Frage, wie ein Gebiet regional aufgegliedert werden muß, damit man zu räumlich relevanten Aussagen kommt. Die verschiedenen Möglichkeiten der regionalen Gliederung für Österreich und ihre je nach Wahl der Bezugseinheit unterschiedliche kartographische Aussagekraft werden in Kapitel 3 dargestellt. An Hand verschiedener Kartenbeispiele soll gezeigt werden, daß die Genauigkeit der dargestellten räumlichen Verteilung umso mehr abnimmt, je stärker die Bezugseinheiten in ihrer Größe und Form innerhalb einer Karte differieren. Eine regionale Gebietsgliederung sollte deshalb möglichst gleich große Bezugseinheiten aufweisen, um Kartenfehlinterpretationen zu vermeiden. 


\section{Zählsprengeleinheiten}

Auf der Basis der Statistischen Zählsprengel sind nur die Großzählungsdaten (VolksHäuser- und Wohnungszählung, Arbeitsstättenzählung) verfügbar. Diese Daten werden in der Regel der ISIS Datenbank (Integriertes Statistisches Informationssystem) entnommen.

Die Zählsprengel stellen in territorialer Hinsicht die kleinsten administrativen Einheiten der amtlichen Statistik dar, über die Daten aus den Großzählungen seit 1971 vorliegen. Über die Hälfte der 2333 Gemeinden sind in Zählsprengel untergliedert, wobei man zwei Arten von Zählsprengel unterscheiden kann:

- Echte Zählsprengel: Um hinsichtlich der Wohnbevölkerung einigermaßen vergleichbare Auswertungs- und Erhebungseinheiten zu erhalten, wurden alle Gemeinden über 2.000 Einwohner in Zählsprengel weiter untergliedert, wobei die durchschnittliche Einwohnerzahl eines Zählsprengels bei 1.000 liegt und zwischen 300 und unter 2.000 schwanken kann. Unter Einhaltung dieser Einwohnerzahlen wurde weiters versucht, bei der Grenzziehung der Zählsprengel auf der Basis der ÖK 1: 50.000 die Struktur der betroffenen Gebiete zu berücksichtigen und möglichst homogene Räume zu schaffen. In ländlichen Gebieten mit im wesentlichen einheitlicher Siedlungsstruktur wurden meist zweckmäßig erscheindende Linien wie Eisenbahnen, Straßen, Wege, Flüsse oder Katastralgemeindegrenzen bei der Abgrenzung herangezogen. Bei Vorhandensein eines Ortes hat man diesen in der Regel von der locker verbauten Umgebung (bzw. den Umgebungssprengeln) getrennt (bzw. den alten Ortsteil vom übrigen Teil der Gemeinde, wenn die Gemeinde nur aus einer geschlossenen Siedlung besteht).

In städtischen Gebieten wurde das Zentrum (ein oder mehrere Zählsprengel) von Wohngebieten verschiedenen Baualters und Bebauungstyps, industriell-gewerblichen Gebieten, landwirtschaftlichen Randgebieten usw. abgegrenzt.

Je nach angewandtem Grenzziehungsmodus gelangt man bei der kartographischen Umsetzung $\mathrm{zu}$ unterschiedlichen, oft nicht direkt miteinander vergleichbaren Ergebnissen.

- Vereinigungssprengel: Zur Erhaltung der Vergleichbarkeit von Territorialeinheiten über längere Zeiträume hinweg wurden alle Gemeinden, die 1961 noch selbständig waren, seither aber mit anderen zu größeren vereinigt worden waren, als Zählsprengel weiter ausgewiesen. Durch Vereinigung von echten Zählsprengelgemeinden mit Vereinigungsgemeinden entstanden Mischgemeinden.

Da man bei der Bildung der Zählsprengel sowohl Verwaltungsgrenzen berücksichtigte, als auch gleichzeitig eine bestimmte Einwohnerzahl anstrebte, weisen die zu vergleichenden Zählsprengel hinsichtlich ihrer Form und Größe oft beträchtliche Unterschiede auf. Dies führte $\mathrm{zu}$ einer Beeinträchtigung der räumlichen Vergleichbarkeit, was an Hand der Kartenbeispiele näher dargestellt werden soll. 


\section{Bezugseinheiten}

Unter Bezugseinheiten versteht man Einheiten, auf die quantitative Angaben, in erster Linie Daten der amtlichen Statistik, ohne genaue Lokalisierbarkeit bezogen sind. Je nach Abgrenzung der Bezugseinheiten kann man zwischen geographischen (z.B. Baublock), administrativen (z.B. Zählsprengel, Gemeinde) und geometrischen (z.B. Quadratgitter) Bezugseinheit unterscheiden, wobei die geometrischen Bezugseinheiten nicht als Alternative zu den administrativen Bezugseinheiten, sondern als diese ergänzende Darstellungsform verwendet werden sollten.

Bei der kartographischen Darstellung der Daten stellt sich auch die Frage nach der Absolut- und Relativwertdarstellung. Bei der absoluten Verteilung werden Mengen und deren Verteilung, bei der relativen werden Relationen zwischen bestimmten Sachverhalten betrachtet. Die Absolutwerte werden im allgemeinen durch flächenproportionel Kreise oder Quadrate, die der Relativwerte durch Flächenraster kartographisch dargestellt.

\subsection{Administrative Bezugseinheiten}

Im System der österreichischen Verwaltungsgliederung stellen die Ortsgemeinden die kleinsten autonomen Selbstverwaltungseinheiten dar. Aus den in Kapitel 2 ausgeführten Gründen hat man die Gemeinden weiters in Zählsprengel untergliedert.

Wie aus der Abbildung $1 \mathrm{zu}$ ersehen ist, variieren die Gemeinden in ihrer Größe und Form sehr stark. Das hat zur Folge, daß bei der kartographischen Darstellung von Sammelsignaturen, gesetzt in den Mittelpunkt einer Gemeinde, sich der tatsächliche Verbreitungsraum der Einwohner nur bei den kleinen Gemeinden annähernd deckt. Zu einem besseren Ergebnis kommt man bei einer Darstellung von Sammelsignaturen, wenn man diese in die optischen Mittelpunkte des Dauersiedlungsraumes (Fläche des Bundesgebietes abzüglich Wald, Almen, Gewässer, Schilfflächen, Ödland) und nicht in die Mitte der Gemeinden setzt (vgl. Abbildung 1 mit Abbildung 2). Ungelöst bleibt aber auch bei dieser Darstellungsform das Problem der Überlagerung der Kreise. Eine Darstellung der Absolutwerte auf der Basis von Zählsprengel ist für eine Österreichkarte im Ausgabemaßstab A4 auf Grund der im Verhältnis zur Darstellung auf Gemeindeebene noch stärkeren Überlagerung der Sammelsignaturen nicht sinnvoll.

In noch stärkerem Ausmaß wird die räumliche Vergleichbarkeit beeinträchtigt, wenn es sich um die kartographische Darstellung von Dichtewerten auf der Basis von administrativen Bezugseinheiten handelt.

Während Österreichkarten auf Gemeindeebene ungeachtet der mangelnden räumlichen Vergleichbarkeit der einzelnene Einheiten auch im Ausgabemaßsstab A4 noch lesbar sind, sind ein Großteil der Zählsprengel in diesem Maßstab nicht mehr klar zu erkennen. Thematische Karten auf der Basis der Statistischen Zählsprengel werden deshalb erst ab einem Maßstab von 1:50.000 und größer sinnvoll, da im innerstädtischen Bereich die Größe der Zählsprengel nicht so stark variiert (vgl. Abbildung 3). Gegen den Stadtrand 
zu werden die Flächen der Zählsprengel allerdings immer größer. Eine Verbesserung der kartographischen Darstellung kann dabei erzielt werden, wenn man den Dauersiedlungsraum innnerhalb der Zählsprengel und nicht ihre Gesamtfläche darstellt. Aber auch diese Darstellung rechtfertigt noch nicht, die Grenzen zwischen unterschiedlichen Dichtewerten auch als räumliche Grenzen zu interpretieren, da die Grenzverläufe vorgegeben sind.

\subsection{Geometrische Bezugseinheiten auf der Basis von Zählsprengelschwerpunkten}

Als Basisfläche geometrischer Bezugseinheiten haben sich Quadrate als günstig erwiesen. Im Rahmen eines Pilotprojektes im Österreichischen Statistischen Zentralamt wurde mit Hilfe des Programms ARC/INFO Österreich mit einem feinmaschigen Quadratgitternetz überzogen. Als ideale Maschengröße hat sich in Versuchen eine Quadratseitenlänge von 2,5km erwiesen, die je nach Ausgabemaßstab leicht auf 5 oder $10 \mathrm{~km}$ vergrößert werden kann. Die Grenzen des Gitternetzes wurden durch das GaußKrüger Netz definiert. Dabei wurde, vom 31. Längengrad östlich von Ferro (M31) ausgehend, das Netz nach Westen und nach Osten über das gesamte Bundesgebiet ausgedehnt. In Nord-Süd Richtung erstreckt sich das Netz von 5130000 bis 5440000 GK und ist damit im Bereich des Mittelmeridians M31 deckungsgleich mit dem Österreichischen Bundesmeldenetz und dem Blattschnitt der Österreichischen Luftbildkarte 1:10.000.

In einem nächsten Arbeitsschritt wurden die optischen Siedlungsschwerpunkte der Zählsprengel digitalisiert und je einer Gitternetzmasche zugeordnet (vgl. Abbildung 4). Anschließend wurden die Einwohnerzahlen der Zählsprengel in jeder Gitternetzmasche aggregiert.

Der große Vorteil bei der Verwendung von Gitternetzen liegt einerseits im übersichtlichen Strukturbild, das sich bei der kartographischen Darstellung des Sachverhaltes ergibt, andererseits können die Dichtewerte der Rastereinheiten bei gleicher Maschengröße unmittelbar miteinander vergleichen werden. Dies ermöglicht auch Vergleiche innerhalb eines Stadtgebietes, aber auch Städtevergleiche und StadtUmland Vergleiche.

Der Nachteil der Gitternetzmethode besteht vor allem darin, daß diese Darstellung sich nur näherungsweise mit dem hierarchischen Aufbau der Verwaltungseinheiten in Beziehung setzen läßt und ein eher abstrakt-geometrisches Bild liefert. Darüber hinaus können die Quadrate zusammenhängende Gebietseinheiten wie Siedlungen oder Baublöcke durchschneiden. Durch Ermitteln von Durchschnittswerten mehrerer beieinander liegender Quadrate kann man aber die Nachteile starrer Bezugseinheiten ausgleichen.

Im allgemeinen wird man aber, zumindest im kleineren Maßstab, den geometrischen Bezugseinheiten gegenüber den Zählsprengeln trotzdem den Vorzug geben, da räumliche und zeitliche Vergleiche mit einem möglichst engmaschigen Gitternetz besser durchgeführt werden können (vgl. Abbildung 5). 


\section{Literatur}

ÖSTERREICHISCHES StATISTISCHES ZENTRALAMT (1987). Die territorialen Grundlagen der österreichischen Bundesstatistik, Wien.

ÖSTERREICHISCHES STATISTISCHES ZENTRALAMT (1989). Graphische und kartographische Produkte, Wien. 132 Seiten.

Desoye, H. (1981). Erläuterungen zu den „Karten der Statistischen Zählsprengel“, Wien, Stand 1981, 2. Aufl. S. 1ff.

WONKA, E. (1993). Computergestützte kartographische Darstellung statistischer Daten mittels Flächensignaturen auf der Basis von geometrischen Bezugseinheiten. In: Berichte und Informationen, Nr. 22, Hrsg. Österreichische Akademie der Wissenschaften, Institut für Kartographie, Wien. 57 Seiten.

Adresse des Autors:

Mag. Martin Weber

Österreichisches Statistisches Zentralamt

Technisch-Methodische Abteilung

Hintere Zollamtsstraße 2b

A-1033 Wien 